\title{
Urbane Metabolismen
}

\author{
Verkörperte Politische Ökologien des e/Essens
}

\begin{abstract}
Anke Strüver
Essen (als Nahrungsmittel) und essen (als Tätigkeit) sind in urbanen Kontexten des Globalen Nordens im Laufe der letzten Dekade zu gesellschaftspolitisch relevanten sowie wissenschaftlich zunehmend beachteten Themen geworden, die als Bestandteile routinisierter Alltagspraktiken auf gesellschaftliche und biosozial verkörperte Ungerechtigkeiten verweisen. Aufbauend auf Sapana Doshis Vorschlägen für eine „embodied urban political ecology“ stellt dieser Beitrag den menschlichen Körper in den Mittelpunkt, um Gesundheits- und Umweltgerechtigkeit anhand des sozio-ökonomisch und -kulturell beeinflussten Zugangs zu gutem Essen sowie der biosozialen Materialisierungsprozesse von essen zu adressieren.
\end{abstract}

Ersteinreichung: 23. Juli 2019; Veröffentlichung online: 24. April 2020

An English abstract can be found at the end of the document.

\section{Einleitung:}

\section{Essen und essen als urbane Mensch-Umwelt-Beziehungen}

Essen (als Produkt) und essen (als Praktik) sind in urbanen Kontexten des Globalen Nordens im Laufe der letzten Dekade zu gesellschaftspolitisch relevanten sowie wissenschaftlich anerkannten Themen geworden (siehe bspw. Morgan 2015; Sonnino 2016; Goodman 2016; siehe auch Tornaghi 2014, 2017 für kritische Zugänge). Essen und essen verweisen zugleich auf routinisierte - und damit im Alltagsgeschehen oftmals unreflektierte - Praktiken auf der räumlichen wie sozialen Mikroebene (del Casino 2015; Evans 2018; Krüger/ Strüver 2018; Warde 2013, 2014, 2016). Ausgehend von dem Zitat „Das, was man in den Körper reintut und dabei der Umwelt antut" aus einem Sample von narrativen Interviews zum Thema ,gutes Essen', rückt dieser Beitrag Essen und essen als Elemente von Routinepraktiken und als Bestandteile urbaner Mensch-Umwelt-Beziehungen in den Mittelpunkt. Dafür werden konzeptionelle Überlegungen aus den Kontexten der feministischen und der Urbanen Politischen Ökologie sowie der Feminist New Materialisms zu biosozialen Verkörperungsprozessen und sozialökologischen Stoffwechselprozessen auf der mikropolitischen Ebene adaptiert und als Ausgangspunkte für die Verschränkungen von Stadt und Ökologie jenseits der aktuell dominanten Wachstums- und technologiefixierten Diskurse zur nachhaltigen Stadtentwicklung diskutiert (vgl. Swyngedouw 2015; Kaika 2017). 
Aufbauend auf Sapana Doshis Vorschlägen für eine „Embodied urban political ecology“ (2017) und der „Political ecology of the body“ von Julie Guthman (2012; siehe auch Guthman/Mansfield 2013 sowie Hayes-Conroy/ Hayes-Conroy 2013, 2015) wird der menschliche Körper in den Mittelpunkt gestellt, um neben dem sozioökonomisch und -kulturell beeinflussten Zugang zu ,gutem Essen“ vor allem die Materialisierungsprozesse von e/Essen[1] zu adressieren. Dies beinhaltet eine Auseinandersetzung damit, wie gesellschaftliche Umweltverhältnisse biosozial verkörpert werden (vgl. Alaimo 2010, 2016; Frost 2016). Dem voran gestellt ist jedoch zunächst eine kurze Auseinandersetzung mit dem Metabolismus-Begriff der Urbanen Politischen Ökologie, da er im weiteren Verlauf dieses Beitrages zwar für die Stoffwechselprozesse zwischen Gesellschaft und Natur beziehungsweise Umwelt angewandt wird, dabei jedoch enger gefasst wird als im Gebrauch der Politischen Ökonomie und Ökologie, nämlich als Stoff-Wechsel zwischen Mensch und Umwelt im wörtlichen und damit im posthumanen Sinne, ohne strukturelle und diskursive Einflüsse auf Essen und essen zu ignorieren. In diesem Denkhorizont umfassen Verkörperungsprozesse also auch die Stoffwechselprozesse, in denen gesellschaftliche Verhältnisse und Diskurse sowie Umweltverhältnisse ,verdaut' werden (Landecker 2011; siehe auch Mol 2008).

Am konkreten Beispiel der Narrationen über ,gutes Essen' lässt sich Stoff-Wechsel thematisieren als stoffliche Umwandlung der Praktiken und Produkte von e/Essen. Weitergeführt wird dadurch die politökonomisch ausgerichtete Urbane Politische Ökologie konzeptionell wie analytisch um Fragen des Zugangs zu ,gutem Essen' und um biosozialen Stoff-Wechsel. In Abschnitt 5 wird dies exemplarisch auf der Grundlage von narrativen Interviews in zwei deutschen Großstädten thematisiert. Dafür wurde in den Interviews sowohl auf die (Eigen-)Definitionen von ,gutem Essen“ im jeweiligen sozialräumlichen Kontext innerhalb einer Stadt (z. B. frisch, regional oder fair produziert, schnell, günstig, gesund, vegetarisch/vegan, biologischorganisch und/oder lokal verfügbar) als auch auf das Tun des essens (z. B. als Notwendigkeit, als Routinepraktik oder als Lust oder Frust) eingegangen.

Ziel der Untersuchung war und ist nicht der Vergleich zwischen Städten oder ihren Bewohner*innen. Das empirische Beispiel dient hier zuvorderst der Illustration und zum Anstoßen einer Diskussion eines Mehrwerts des konzeptionellen Ansatzes Verkörperter Urbaner Politischer Ökologien unter den zunehmend ungerechten sozialen und ökologischen Bedingungen im Kapitalozän und Anthropozän (Haraway 2016: 30 ff.). Konkret geht es im Kontext dieses Beitrags erstens darum, die Auseinandersetzungen mit dem sozioökonomisch und -kulturell beeinflussten Zugang zu ,gutem Essen“ (siehe bspw. Augustin 2014) um die materielle Ko-Produktion von Körpern und urbaner Umwelt im Sinne biosozialer Verkörperungen beziehungsweise transkörperlicher Relationen zu erweitern. Essen und essen als biosoziale Prozesse zu fokussieren, beinhaltet zweitens die Möglichkeit, die abstrakten ontoepistemologischen Überlegungen zur Auflösung der Dualismen von Kultur und Natur sowie von Mensch und Umwelt von beispielsweise Donna Haraway (2016) und Karen Barad (2015) anhand eines Alltagbeispiels (e/Essen) gesellschaftstheoretisch und sozial-ökologisch zu explizieren. Drittens macht das Beispiel es methodologisch und methodisch erforderlich, 
sich auf das Experimentieren mit more-than-human und more-than representational Methodologien einzulassen, die eher Relationen fokussieren als Fakten präsentieren.

\section{Urbane Politische Ökologien}

Seit der Jahrtausendwende wurde immer stärker auf die Leerstellen in der Auseinandersetzung mit lokalen sozialökologischen Perspektiven in urbanen Räumen hingewiesen. Heynen et al. (2006) haben daraufhin ein Manifest für eine Urbane Politische Ökologie (UPE) skizziert, das soziale und ökologische Gerechtigkeitsfragen mit den Prinzipien der kapitalistischen Stadtentwicklung konfrontiert. Dafür greifen sie unter anderem Marx' Metapher des Metabolismus auf, um die Beziehungen zwischen Kultur und Natur, zwischen Mensch und Umwelt als Stoffwechsel zu adressieren, als „change of matter [that] [...] simultaneously implies circulation, exchange and transformation of material elements" (Swyngedouw 2006: 26; Herv. i. O.).

Das Manifest betont die Wechselwirkungen zwischen sozialer und ökologischer Umwelt als metabolische Prozesse unter expliziter Berücksichtigung der Frage gesellschaftlicher Machtverhältnisse (Heynen et al. 2006: $11 \mathrm{ff}$.). In zahlreichen empirischen wie konzeptionellen Aus- und Weiterführungen dieser UPE wurde unter anderem der Greening-Anspruch der dominanten Nachhaltigkeitsdiskurse kritisiert, die eher technologieorientiert und -optimistisch als grundsätzlich kapitalismus- und gesellschaftskritisch funktionieren und die zwar mehr Grün (,Natur') in der Stadt einfordern, aber eine grundsätzliche Auseinandersetzung mit der Kommodifizierung von (urbaner) Natur vernachlässigen (Kaika/Swyngedouw 2011; Swyngedouw/Kaika 2014): "[S] ustainability' became the empty signifier that referred generically to the phantasmagoric vision of a world in which people, the economy and the environment could happily and lovingly interact in mutually supportive, cohesive, and historically reproducible manners, mediated by increasingly ,smart' technologies that would benignly micro-engineer the delicate balance between humans and nature." (Swyngedouw/Kaika 2014: 467).[2]

Von der UPE eingefordert wird daher eine erweiterte Betrachtung von Ressourcen- und Umweltgerechtigkeit einerseits und des sozialen wie ökologischen räumlichen Kontexts auf der lokalen Ebene andererseits - ohne die globale Dimension des übermäßigen und ungleich verteilten Ressourcenverbrauchs durch städtisches Leben und städtisches Wachstum aus den Augen zu verlieren. Im Fokus steht somit die Politisierung von urbaner Natur im Sinne ihrer sozioökonomischen Kommodifizierung und ihrer Potentiale für eine sozialökologische Transformation, einschließlich eines Verständnisses von Metabolismus, in dem „the matter of matter becomes an active moment in the political-ecological transformations that shape planetary urbanization" (Swyngedouw 2015: 612).

Der UPE-Anspruch „to re-nature the city“ und „to re-nature urban studies“ (Kaika/Swyngedouw 2011) hat trotz der Stoffwechsel-Metapher bislang urbane Ernährung im globalen Norden weitgehend ignoriert (vgl. Galt 2013). Dies wird im vorliegenden Beitrag in dem Sinne aufgegriffen, e/Essen als Thema des Urbanen stärker einzuführen und entlang einer neuen Perspektive zu diskutieren. Denn auch in den Food Studies wird anerkannt, 
dass „the ways in which food is accessed, produced, and consumed in cities are entangled in the socionatural production of urban space at different scales (...): body, home, city, and beyond" (Shillington 2013: 104; siehe auch Morgan 2015; Sonnino 2016). Vor diesem Hintergrund fokussiert der Beitrag die Verkörperungen der Verschränkungen zwischen Gesellschaftlichem und Ökologischem und stellt expliziter die Frage „how matter comes to matter“ (Barad 2003; Hervorh. A.S.).

\section{Feministische Politische Ökologien und Posthumane Materialisierung}

Während die (neo-)marxistische UPE gesellschaftliche Strukturen und Ungleichheiten fokussiert, schlagen feministische Ansätze aus dem Kontext der New Materialisms und der Verkörperten (U)PE vor, sozial- beziehungsweise stadträumliche Gerechtigkeiten erstens intersektional und zweitens noch stärker ökologisch (bzw. biosozial und sozialökologisch) zu thematisieren - und damit einhergehend den Körper- sowie den Stoffwechselbegriff viel konkreter zu adressieren.

Feministische Politische Ökologien berücksichtigen Geschlechterverhältnisse in Relation zur ökologischen Umwelt auf unterschiedlichen Maßstabsebenen (Elmhirst 2015). Umwelt wird dabei, ausgehend vom etablierten feministischen Fokus auf sozialstrukturelle, kulturelle und diskursive Dimensionen, zurückgeführt aufökologische Umwelt als materielles Substrat, das mit Lebewesen in einer Wechselbeziehung steht. Im Unterschied zur klassischen und auch zur Urbanen Politischen Ökologie werden zudem vor allem die Wechselwirkungen zwischen ,Natur' und ,Kultur' als Teil von Verkörperungsdynamiken in den Blick genommen (vgl. Hawkins et al. 2011). Voraussetzung dafür ist ein Überkommen der ontologischen Grenzen zwischen Natur und Kultur, Umwelt und Mensch, eine posthumane relationale Ontologie, wie sie im Feminist New Materialism entwickelt worden ist (vgl. Alaimo/ Hekman 2008; Alaimo 2016; Barad 2003, 2007; Frost 2016; Haraway 2008; siehe auch Heynen 2018). Dadurch wird das Naturverständnis erweitert und es geht nicht nur um gesellschaftlich konstruierte Naturvorstellungen und gesellschaftliche Naturverhältnisse beziehungsweise Ressourcen(aus)nutzungen, sondern auch um Natur als Materialität, die in den Körpern von Lebewesen aktiv ist. Am konkreten Fall der Ernährung umfasst das die menschliche Aktivität des essens (Praktik) und die Aktivität des Essens (Materialität) im Körper - ohne normierende Ernährungsdiskurse und gesellschaftliche Machtverhältnisse zu ignorieren. Im Mittelpunkt der Betrachtung steht dann die Erweiterung der Frage nach der Inkorporierung gesellschaftlicher Strukturen und Diskurse um Verkörperungen ökologischer und sozioökonomischer Umweltverhältnisse.

Um die Auseinandersetzung mit der Materialität gesellschaftlicher Umweltverhältnisse voranzutreiben und stofflich-materielle Prozesse in sozialund kulturwissenschaftliche Analysen einzubeziehen, eignen sich Haraways radikal-relationale Konzeption von Naturecultures (v. a. 2008, 2016; siehe auch 1995, 2000) und Barads Ansatz der Posthumanist Performativities (v. a. 2003, 2007, 2010; auch 2012, 2015) als gesellschafts- und erkenntnistheoretische sowie ontologische Grundlagen. Beide Autorinnen sind in 
der feministischen Naturwissenschafts- und Gesellschaftskritik verortet und haben wichtige Überlegungen für die (Feminist) New Materialisms geliefert. Beide Ansätze eint zudem die Absicht, Denaturalisierungs- mit Dekulturalisierungsstrategien zusammenzubringen, um jenseits von biologischen Determinismen oder kulturellen Essentialismen die response-ability (Haraway 2016: 115) und Intra-aktivität (Barad 2015) zwischen Sozial-Diskursivem und Biologisch-Materiellem als transkörperliche Relationen sowie als biosoziale Verkörperung offenzulegen. Verkörperung wird dann nicht nur durch gesellschaftliche Strukturen und Diskurse, individuelle Wahrnehmungen und Praktiken konstituiert. Vielmehr sind auch materielle Prozesse einflussreich, die durch Intra-Aktionen zwischen Natur und Kultur, zwischen Biologischem und Sozialem den menschlichen Körper produzieren.

Die Debatten der Feminist New Materalisms haben eine Konzeption von menschlichen Körpern als gleichzeitig diskursiv konstituierte, politisch umkämpfte und materielle Orte eingeführt -die also zugleich durch die sozialen und ökologischen Verhältnisse produziert werden. Dennoch werden diese Debatten im Kontext der UPE bislang theoretisch wie empirisch (zu) selten berücksichtigt (siehe Bauriedl 2016 für eine konzeptionelle Übersicht). Sapana Doshi (2017: 126f) hat fünf Vorschläge für eine Verkörperte Urbane Politische Ökologie formuliert, die einige der aktuellen feministischen Ansätze zusammenführt und die hier - zum Teil neu sortiert - kurz umrissen werden:

1. (5) Körper sind die Orte, an denen politische Subjekte geformt werden. Diese Feststellung war und ist zentraler Bestandteil der feministischen Argumentation zur Körperkonstitution (vgl. Strüver 2012).

2. Fragen der sozialen Reproduktion sind wesentlicher Bestandteil urbaner politischer Ökologien. Ohne die oft vernachlässigten und lästigen, schmutzigen und körperlichen Elemente sozialer Reproduktionsarbeiten mitzudenken, können kapitalistische Stadtentwicklungsprozesse nicht umfassend kritisiert werden (vgl. Federici 2015; Schuster/Höhne 2017).

3. Machtverhältnisse sind relational und intersektional. Es bedarf eines Fokus darauf, wie vergeschlechtlichte, rassifizierte und sozioökonomisch strukturierte Machtverhältnisse räumlich operieren und wie verschiedene Marginalisierungs- und Exklusionsmechanismen je nach räumlichem Kontext unterschiedlich erfahren und wahrgenommen werden (vgl. Mollett/Faria 2013, 2018).

4. Affekte und Emotionen sind Teil der materiellen Verkörperung von Subjekten. Sie lassen sich viszeral und im Zusammenwirken mit diskursiven und strukturbedingten Verkörperungen adressieren: ,how visceral experiences - a relational field of emotion and corporeality - connect with structural and discursive spheres“ (Doshi 2017: 127 f.; s.u.).

5. (1),Metabolismus' ist nicht nur metaphorisch interessant, sondern auch als verkörperte Politik relevant: Der Begriff setzt sozioökologische Produktions- und Konsummuster sowie Ressourcenverbrauch in direkte Beziehung zur Intimität machtgeladener Verkörperungen.

Für gesellschaftstheoretische wie -praktische Überlegungen zur biosozialen Materialität von Verkörperungen betonen Hayes-Conroy/Hayes-Conroy $(2013,2015)$ das Viszerale als Bestandteil gesellschaftlicher Strukturen und Diskurse, um zu analysieren, wie das Sozioökonomische und -kulturelle sich 
biologisch materialisieren (siehe auch Guthman 2012; Guthman/Mansfield 2013). Sie skizzieren eine Political Ecology of the Body (PEB), die am konkreten Beispiel der Ernährung unter anderem analysiert, inwiefern ,gutes Essen' gleichzeitig sozial, materiell und emotional ist und daher im Rahmen einer posthumanen relationalen Ontologie auch weiterhin sozioökonomische Strukturen und kulturelle Diskurse berücksichtigt werden müssen. In ihren eigenen empirischen Forschungen (Hayes-Conroy/HayesConroy 2013, 2015) arbeiten sie heraus, dass beispielsweise die lokale Nahrungsmittelverfügbarkeit das Gefühl für ,gutes Essen ' beeinflusst, genauso wie soziokulturelle Gewohnheiten und gesellschaftliche Diskurse über gesunde und/oder nachhaltige Lebensmittel. Sie schlussfolgern, dass der Aspekt des Zugangs - jenseits der üblichen Konzentration auf Angebot und Nachfrage - stärker einbezogen werden müsse.[3]

Eine Verkörperte Politische Ökologie bringt strukturelle Ungleichheiten mit dem (Zugang zu) Wissen über ,gutes Essen', viszeral-emotionalen Erfahrungen und biosozialen Mechanismen als posthumane beziehungsweise transkorporeale Intra-aktivitäten zusammen (Hayes-Conroy/HayesConroy 2015: 662 f.; siehe auch Mol 2008, 2009): Sie ist in der Schnittmenge dieser drei Dimensionen angesiedelt, da beispielsweise der räumliche und sozioökonomische Zugang und der soziokulturelle Kontext das Erleben von Geschmack, aber auch die biosozialen Materialitäten beeinflussen kann. Zugleich haben familiäre Alltagsroutinen und Wissenskontexte Einfluss darauf, was als ,lecker', als gesund oder leicht verfügbar, was als ,gutes Essen' gilt.

\section{Verkörperungs- und Stoffwechselprozesse}

Die Verkörperung von ökologischer Umwelt als transkörperliche Relationen lässt die sozioökonomischen Umweltverhältnisse sowie Diskurse, die als Rassismen, Klassismen und Sexismen wirken, nicht außer Acht. Sie bedarf als Analyseperspektive gleichwohl der Auflösung der epistemologischen und ontologischen Grenzen zwischen Ökologischem und Gesellschaftlichem, Natur und Kultur, Umwelt und Körper - und einer posthuman gerahmten Definition von Wechselwirkungen und Stoffwechselprozessen. MenschUmwelt-Beziehungen werden somit zu Naturkultur-Wechsel-Wirkungen (Haraway 2016); sie basieren auf materiell-diskursiven Verschränkungen, auf intra-aktiven Praktiken zwischen Mensch und Umwelt (Barad 2015; Alaimo 2016). Megan Warin (2015) hat vor diesem Hintergrund eine biosoziale Perspektive auf Verkörperungen anhand der Verschränkungen von biologischer Materialität, sozioökonomischer Realität und Ernährungsbeziehungsweise Gesundheitsdiskursen am Beispiel von Ernährung und Körpergewicht skizziert. Einmal mehr wird dafür die Auflösung der epistemologischen und ontologisch fundierten Trennung und akademischen Arbeitsteilung zwischen biomedizinischer, naturwissenschaftlicher Forschung und sozial- beziehungsweise kulturwissenschaftlichen Ansätzen gefordert, um relationales Denken stärker auf die Relationen und Intraaktionen zwischen Sozialem und Biologischem - auf Grenzbeziehungen statt auf Grenzziehungen - anwenden zu können.

Abstrakter spricht Jamie Lorimer (2017) von Mensch-Umwelt-Beziehungen als more-than-human oder multispecies Ontologie und von einem 
,probiotic turn', der die Gesundheit der Menschen mit der der ökologischen Umwelt auf Basis einer gemeinsamen sozialökologischen Ontologie zusammenbringt (siehe auch Haraway 2008, 2016 sowie Gesing et al. 2018; Romero et al. 2017; Strüver 2019). In einer solchen posthumanen Rahmung wird die konstruktivistische Frage nach der soziokulturellen Wirkmächtigkeit biologischer Unterschiede um eine biosozial-materielle Frage nach den biologisch-verkörperten Effekten sozioökonomischer und -kultureller Ungerechtigkeiten ergänzt (Marquardt/Strüver 2018). Diese Erweiterung berücksichtigt, dass die Ursachen für den unterschiedlichen Zugang zu und das Wissen über ,gutes Essen` auf sozioökonomische und -kulturelle Ungleichheiten zurückgehen, deren Folgen aber auch biophysische sind. Dies wird am Beispiel der Praktiken und Materialitäten von ,gutem Essen“ kurz illustriert.

\section{Transkorporeale Intra-Aktivitäten: Essen und essen}

Sich auf Essen und essen als urbane Mensch-Umwelt-Beziehungen und als Teil einer verkörperten Politischen Ökologie zu konzentrieren umfasst neben dem sozioökonomisch und -kulturell beeinflussten Zugang zu ,gutem Essen die biosoziale Materialisierung von e/Essen. Da in deutschen Großstädten die Nahversorgung mit Lebensmitteln in Wohnungsnähe als gut und gesichert gilt (siehe bspw. Anders 2015; Augustin 2014), beziehen sich Unterschiede im räumlichen Zugang eher auf die Art des Angebots und die Auswahl (d. h. welcher Supermarkt und/oder Discounter) als über Segregationsmuster durch sogenannte food deserts wie in den USA (für eine kritische Reflektion, siehe Shannon 2014). Nicht zuletzt deswegen spielen die unterschiedlichen Wahrnehmungen und Bewertungen von ,gutem Essen' eine Rolle.

Um e/Essen als zentralen Bestandteil von Mensch-Umwelt-Beziehungen im biosozialen Sinne zu adressieren, dient das nachfolgende empirische Beispiel dem Anstoßen einer Diskussion zu Verkörperten Urbanen Politischen Ökologien unter sozial und ökologisch ungerechten Umweltbedingungen. Es besteht zum einen aus der inhaltsanalytischen Zusammenfassung von 29 ,Eat-Along'-Interviews in Hamburg und Berlin zum Thema ,gutes Essen“ (in Anlehnung an Carpiano 2009). Im Kontext dieses Beitrages hat das Beispiel zum anderen zum Ziel, die (Selbst-)Wahrnehmung des sozioökonomisch und -kulturell beeinflussten Zugangs zu ,gutem Essen“ um biosoziale Verkörperungen im Sinne transkörperlicher Umweltrelationen als postdualistische Konzepte zu erweitern.

Die Interviews sind im Kontext des Forschungsprojekts „Relationale Geographien der Ernährung im Spannungsfeld zwischen Privatsache und Politikum" entstanden.[4] Sie wurden zwischen 2016 und 2018 in Hamburg und Berlin geführt, zunächst auf Basis der digitalen Mitschnitte transkribiert und als Teil einer strukturierten Qualitativen Inhaltsanalyse deduktiv kodiert und ausgewertet (vgl. Mayring 2014).[5] Das Kategoriensystem umfasst unter anderem (1) Definitionen von ,gutem' Essen (u. a. gesund, günstig, lecker, regional, ökologischer Anbau, leicht verfügbar im Sinne von zeitlicher oder räumlicher Erreichbarkeit, fair produziert etc.) und (2) Aspekte des Alltagslebens, die ,gutes Essen ' befördern oder behindern (bspw. familiäre und kulturelle Gewohnheiten, finanzielle (Un-)Möglichkeiten, 
Mobilitätsroutinen, Verantwortungsbewusstsein - für die soziale oder ökologische Umwelt, die eigene Gesundheit oder die der Familie -, Lustund Frust-Food etc.)[6]. Für diesen Beitrag zur Verkörperten Politischen Ökologie in Anlehnung an Guthman (2012), Hayes-Conroy/Hayes-Conroy (2013, 2015) und auch $\mathrm{Mol}(2002,2008,2009)$ fand zudem eine zusätzliche ,Anhörung' des Interviewmaterials statt, um aufbiosoziale Materialisierungen als Teil von mikropolitischen Mensch-Umwelt-Relationen zu fokussieren (s. u.). Das heißt, mit rückwirkendem Blick auf die Interviews ist im Kontext dieses Aufsatzes insbesondere von Interesse, ob und wie biosoziale (und damit relationale) Phänomene wie Verkörperungen durch Routinepraktiken des essens thematisiert und wahrgenommen werden.

\subsection{Räumlicher, soziokultureller und ökonomischer Zugang zu,gutem Essen‘}

Den Einstieg in die Interviews bildete die erzählgenerierende Frage nach ,gutem Essen' (Produkt) im eigenen sozialräumlichen Kontext. Im weiteren Verlauf konzentrierten sich die Gespräche vor allem auf die Routinepraktiken des essens, zum Beispiel als alltägliche Notwendigkeit, als fixes Familienritual, aus Geselligkeit, als Teil der personalen Identität. Dies beinhaltete oftmals direkt Widersprüche zur Einstiegsfrage zur Charakterisierung von ,gutem Essen' als Produkt. Dadurch wird deutlich, dass erstens gutes Essen leichter zu definieren als zu praktizieren ist und dass zweitens häufig zwischen allgemeinen Ansprüchen und eigenem Alltagsleben unterschieden wird. Alle Interviewten betonen, wie sehr ihre Essenspraxis mit den eigenen Idealen beziehungsweise Definitionen von ,gutem Essen“ kollidiere. Die Gründe dafür finden sie sowohl in Aspekten der räumlichen Rahmenbedingungen (Angebot und Auswahlmöglichkeiten in räumlicher Nähe zu Wohnung oder Arbeit; oftmals auch in Kombination mit, nah und schnell'), als auch in den zeitlichen und räumlichen Alltagsroutinen (z. B. Familie, Arbeit, Freizeit) sowie individueller Lust (und Frust) am e/Essen.

Der räumliche Zugang hat eine große Bedeutung und die Interviewten erzählen, dass ihre alltägliche Ernährung stark durch das lokale Angebot an und in Supermärkten, Discountern und Biomärkten bestimmt wird, wie etwa: „Für zu Hause geh ich meistens im REWE einkaufen; das hängt aber auch damit zusammen, dass es in der Nähe ist. Aber eigentlich bin ich ein EDEKA-Käufer, weil dort find' ich das Gemüse von der Qualität ein bisschen besser“(HH 24). Noch konkreter berichtet ein Rentner in Hamburg (HH 19): „Am wichtigsten ist die Nähe, dass ich da zu Fuß hinkomme. Lieber jeden Tag zum Penny als einmal die Woche mit dem Bus zum Einkaufszentrum, wo es die größere Auswahl gibt.“

Finanzielle(Un-)Möglichkeiten sind thematisch in allen geführten Interviews präsent - und zwar ohne diesbezügliche Fragen/Nachfragen. Auffällig ist dabei, dass finanzielle Machbarkeiten gleichermaßen von Prekarisierten wie von Gutsituierten angesprochen werden - und dass finanzielle Aspekte jenseits von eindeutiger Einschränkung oder Ermöglichung als allgegenwärtig, quasi selbstverständlich akzeptiert werden. Die Bereitschaft, für (gutes) Essen angemessen zu bezahlen ist daher ambivalent, oft sogar innerhalb einer Person: „Gut ist das, was günstig ist. Ja, das klingt wie in 
der Werbung, ist aber so. ... Obwohl ... gesund ist das wohl nicht, dass ich immer auf den Preis achten muss." (HH 16) Es finden sich aber auch direkte Bezüge auf finanzielle Einschränkungen, die diese selbstverständlich in direkte Beziehung zu räumlicher Nähe und Qualität (,frisch') setzen: „Wenn es schnell gehen muss, geh ich bei uns um die Ecke zu NETTO, obwohl ich NETTO hasse, weil das immer so vollgestopft ist und man gar kein Überblick hat; wirklich nur wenn's schnell gehen muss und ich keine Lust hab zu LIDL zu fahren. Aber sonst bin ich mit LIDL, mit dem Angebot und den Preisen sehr zufrieden. Und Fleisch hol ich vom Türken um die Ecke, weil das einfach täglich frisch ist." (B 26)

Ein anderer Punkt sind familiäre und kulturelle Bezüge, die das e/Essen beeinflussen, und die sich in drei Aspekte differenzieren lassen: (1) „Ich hab türkische Wurzeln, das prägt, was ich esse und mit wem ich esse. Essen ist ein Familiending“ (B 3) und (2) „Hauptsache, es ist für die Kinder gut“ (ebd.). Ganz anders sind hingegen (3) ,ich bin Nachkriegskind, ich weiß mir immer zu helfen“ (HH 22) und „früher bei uns auf dem Land war das nie Thema: Du guckst, was da ist und das kommt auf den Tisch; wenn nichts da ist, also wenig, musst du improvisieren. Ich hab das irgendwie verinnerlicht: Ich hab Spaß am Improvisieren, das ist viel erfüllender als noch spät abends zum Rewe zu gehen... [lacht wegen, erfüllend'] ... geht es um Auffüllen oder Erfüllen? Ich glaube, heutzutage verwechseln viele Auffüllen mit Erfüllen ... und deswegen wird auch so viel verschwendet." (HH 20)

In der Gesamtanalyse wird deutlich - trotz und zugleich aufgrund des explorativen Charakters -, dass insgesamt, gutes Essen' gleichermaßen und gleichzeitig sozioökonomisch wie kulturell, materiell und emotional-viszeral sein kann und dass sowohl die leichte Verfügbarkeit beispielsweise im Sinne von räumlicher Nähe oder schneller Lieferung beziehungsweise Zubereitung, unhinterfragte Alltagsroutinen, aber auch spontaner Appetit (v. a. ausgelöst durch Essensdüfte und Werbung u. ä.) in hohem Maße ausschlaggebend für die konkrete Lebensmittelwahl und -konsumption sind.

\subsection{Diffraktion als subtile Version von Reflexion}

Eine posthuman inspirierte Analyse der Routinepraktiken des e/Essens vor dem Hintergrund der Wechselwirkungen zwischen Mensch und Umwelt beziehungsweise Kultur und Natur basiert auf der Aktivität des essens (als Teil menschlicher Praktiken) und der Aktivität des Essens (als ,Ding“, als nicht-menschliche Materialität) - beide Aktivitäten sind in ihrer IntraAktivität elementar für Verkörperungsprozesse. Eine derartige Analyse bedarf auch einer posthumanen Methodologie, die die Kriterien quantitativer und qualitativer Methoden hinterfragt. Sie ist als Weiterführung kritischer Forschung zu Ungleichheiten einzuordnen, die den Aspekt der Gerechtigkeit gleichermaßen mikro-politisch wie planetarisch adressiert (vgl. Ulmer 2017; Braidotti 2019). Zugleich beinhaltet der Anspruch auf posthumane Relationalitäten die Schwierigkeit, humanistische und (post-)strukturalistische Ansprüche auf Verantwortung, Veränderung und Gerechtigkeit beizubehalten: „more-than-critical methodologies are needed for a more-than human world" (Ulmer 2017: 834). Dies führt zu mehreren Erweiterungen, erstens des Verantwortungs- und Gerechtigkeitsanspruches 
auf das Mehr-als-Menschliche des Lebens (z. B. Pflanzen, Tiere, Böden, Wasser), zweitens des Fokus auf biosoziale Intra-Aktionen, auf mehr-alsmenschliche Verkörperungsprozesse, und drittens des dezentrierten Subjekts. Viele Autorinnen der Feminist New Materialisms greifen hier erneut (bzw. immer noch) auf Haraways Konzept des Situierten Wissens (1995) zurück, um gleichzeitig kritisch wie ,unvollständig' zu forschen - ,partiell gilt hier als das positive Andere von ,universell ${ }^{\star}$-, und um das Beforschte als offenen sozialen wie materiellen Prozess zu akzeptieren (siehe auch Alaimo 2016; Barad 2007, 2014; Braidotti 2019 sowie Fox/Aldred 2018; Lenz Taguchi/Palmer 2013). Als Teil des Konzepts des Situierten Wissens hatte Haraway bereits früh das Positionieren gegenüber dem Reflektieren eingeführt (1992, 1995, 1997), da Letzteres an der Suche nach dem „really real“ (1997: 16) festhält - und scheitert. Sie schlägt ,Diffraktion als subtile Version von Reflexion'vor - als Methode eines kritisch situierten Bewusstseins, das sich vor allem auf das relationale Wesen von Grenz(be)ziehungen konzentriert - und dies auch im Sinne einer Sorge um Gerechtigkeit praktiziert (ebd., siehe auch Haraway 2016). Darauf aufbauend basiert für Barad (2007: 72, 86 ff.) situiertes Wissen auf dem dreigeteilten Arrangement von Forschenden, Forschungsobjekten und Forschungsrepräsentationen (z. B. Interviewmitschnitten), das heißt die Forschenden sind nicht Außenstehende, sondern Teil der Relationen und Reflexionen - und Diffraktionen werden zu mehr-als-repräsentationalen, performativen Werkzeugen, die Wissen, Denken, Messen und Beobachten als intra-aktive Praktiken in Abgrenzung zum Auffinden von Fakten konzeptionalisieren.[7]

Diffraktion als performative Praktik in der Wissensproduktion kann für den Umgang mit Interviews bedeuten, dass weniger die transkribierten Texte als Kodier- und Interpretationsgrundlage berücksichtigt werden müssen und mehr ein Einfühlen in die Forschungssituation gefordert ist. In Anlehnung an Hillevi Lenz Taguchi (2012; sowie konkret Barad 2007: 89 und Bozalek/Zembylas 2017: 119 f.) werden daher für Diffraktion als subtile Reflexion im zweiten hier präsentierten Analyseschritt nur die Interviews berücksichtigt, die von anderen Mitgliedern des Forschungsteams geführt wurden; zudem wurden anstatt einer Verwendung der Text-Transkriptionen die Originaldateien angehört. Dies stellt auch den Versuch dar, den Anthropozentrismus qualitativen empirischen Arbeitens zumindest im Hinblick auf rational-kognitive und phänomenologische Ansätze zu hinterfragen und für eine Hinwendung zu verkörperten Methoden zu plädieren, die gleichwohl das Denken nicht ignorieren, sondern die Forschungssubjekte als „embodied, embrained, relational, affective“ (Braidotti 2019: 51) akzeptieren. Das heißt, abgelehnt wird der Anspruch auf eine logozentristische und universalistische Wissensproduktion. Ersetzt wird er durch eine Situierung, die als embodied and embrained viel mehr als eine reine Subjektivierung darstellt; Denken ist nicht individuell und subjektiv, sondern im epistemologischen wie ontologischen Sinne,weltlich“ (Alaimo 2014; Braidotti 2019).

\subsection{Biosoziale Verkörperungen durch e/Essen}

In den Erzählungen zu ,gutem Essen“ wird dieses häufig mit gesunder Ernährung gleichgesetzt, oder mit Attributen beschrieben, die gemeinhin als 
gesund gelten. Gemeinsamer Nenner dieser Attribute ist ,frisch - individuell wird darunter frisches Gemüse, frisches Fleisch oder auch einfach ,frisch zubereitet' verstanden. Gutes Essen kann aber auch das sein, was für wenig Geld beziehungsweise mit wenig Zeitaufwand satt macht, oder Essen, das auf Weglassen oder Vermeiden basiert, etwa: „Dieser Hype ist ja auch erst gekommen mit laktosefrei und glutenfrei usw. Der Frei-Hype und die ganzen Ersatzprodukte ... und die Menschen kaufen es, weil sie sagen okay, das ist gesund!“ (HH 24) Bei all diesen Auslegungen wird Verantwortung primär für den eigenen Körper übernommen. Aussagen wie die folgenden stellen dagegen rare Ausnahmen dar: „Als Konsument hat man auch Verantwortung gegenüber der Umwelt und den Verkäufern oder Verkaufsstrukturen. [...] Ich glaube auch, dass Bio-Lebensmittel größtenteils vielleicht gar nicht so viel besser für mich sind, aber unter vernünftigeren Produktionsbedingungen entstanden sind.“ (HH 19) Noch viel stärker betont dies $\mathrm{HH}_{3}$, indem sie „das [kritisiert], was man in den Körper reintut und dabei der Umwelt antut“ und dadurch implizit auf die Wechselwirkungen zwischen menschlichen Körpern und ökologischer Umwelt im Sinne biosozialer Verkörperungen beziehungsweise transkörperlicher Relationen verweist. Ein Veganer aus Hamburg (HH 17) interpretiert sich und sein Leben in diesem Wissenskontext genau andersherum: „Ich lebe vegan, fair und günstig, da ich containern gehe. Das ist mir eine gerechtere Welt wert, auch wenn es vielleicht nicht immer das Gesündeste für mich ist.“

Direkt thematisiert wird das Biosoziale zudem in Aussagen wie diesen: „Eigentlich bin ich so eingestellt, dass ich versuche mich immer gesund zu ernähren; aber wenns denn knapp ist, dann guckt man okay, wie geht das jetzt noch zu vereinbaren und da muss ich Abstriche machen. Aber das Schlimme ist halt, dass man dann auch die Nachteile direkt spürt, wenn man wirklich schlechteres und verarbeitetes Essen isst." (HH 24) Ähnlich - auch im Hinblick auf den Stoffwechsel - beschreibt es B 26: „Es sei denn, wir sind faul und bestellen Essen, das machen wir sehr ... zu viel. Also für die 30 Euro Jumbopizza mit den 20.00o Zutaten hätte ich auch für 30 Euro zwei Wochen Grundnahrungsmittel kaufen können. Der Müll, den hast du dann gegessen; fühlst dich zwar voll und träge und denkst oha toll, du bist satt. Nach zwei Stunden hast du aber wieder Hunger, weil da wirklich nur Schrott drin ist. Und am nächsten Tag hast du erst mal schön [lacht] auf der Toilette zu tun." Eine weitere Dimension transkörperlicher Relationen wird hingegen in Aussagen wie diesen offenbar: „Der [Obstbauer] ist komplett bekleidet mit weißer Kleidung und hat Atemschutz und pflückt den Apfel und schmeißt ihn in den Pott und dann wird das gewaschen und verkauft und dann heißt es, oha aus der Region, oha, das ist aber bestimmt gesund?“(B 26) Und wiederum genau andersherum beschreibt es $\mathrm{HH}$ 28: „Ich finde regional manchmal sogar wichtiger als Bio, so dass es halt irgendwie aus der Ecke kommt und dass ich drauf achte. Ich hab irgendwie das Gefühl, dass es mich dann mehr mit dem Essen verbindet." Im zweiten Analyseschritt mit dem Fokus auf transkörperliche Relationen und biosoziale Intra-Aktivitäten deutet sich - ungeachtet des experimentellen Charakters - an, dass die Reflexionen über die Routinepraktik essen dieses als gleichermaßen gesellschaftlich und körperlich, kulturell und materiell-stofflich wahrnimmt. 


\section{Verkörperte Politische Ökologien in der Stadt}

„How to feed cities [in the Global North] in a just, sustainable and culturally appropriate manner in the face of looming climate change, widening inequality and burgeoning hunger is one of the quintessential challenges of the 21st century."(Morgan 2015: 1390) Die hier präsentierte ontoepistemologische Diskussion und die empirische Illustration zu urbanen Metabolismen des e/Essens ermöglichen erstens, die Kultur-Natur- und Mensch-Umwelt-Dualismen aufzubrechen und stattdessen ein radikal-relationales und posthumanes Denken anzuwenden; zweitens, e/Essen als Elemente urbaner transkorporealer Mensch-Umwelt-Beziehungen zu fokussieren und damit Stadt und Ökologie auf der Mikroebene von Routinepraktiken theoretisch wie praktisch enger zusammen zu bringen; und drittens, eine Diskussion zur Verkörperten Politischen Ökologie anzustoßen, die sich auch auf transkörperliche Umweltrelationen und biosoziale Verkörperungen konzentriert.

Die aus der feministischen UPE und den New Materialisms abgeleiteten Überlegungen zu biosozialen Verkörperungen und sozialökologischen Stoffwechselprozessen erlauben, more-than-human Verschränkungen auf der Mikroebene zu fokussieren und neben Fragen des sozioökonomischen und -kulturellen Zugangs zu ,gutem Essen' den Begriff des Stoff-Wechsels wörtlicher zu nehmen: als „change of matter“ (Swyngedouw 2006: 26). Essen und essen als biosoziale Prozesse zu thematisieren bietet damit eine Perspektive, um die Verschränkungen von Körpern und Umwelt als transkörperliche Relationen zu adressieren. Das heißt, Stoffwechsel ist auch als Verkörperungsprozess relevant, da ,gutes Essen' gleichzeitig sozial, materiell und emotional ist - und beispielsweise das lokale Angebot das Gefühl für ,gutes Essen' genauso beeinflusst wie Gesundheits- und Nachhaltigkeitsdiskurse und alltägliche Routinen.

Die Zusammenfassung der Ergebnisse des empirischen Beispiels zu ,gutem Essen' macht zudem deutlich, dass in den Selbstwahrnehmungen und -beschreibungen von ,gutem Essen 'Verweise auf die sozialräumlichen und soziokulturellen Kontexte sowie die Ambivalenzen zwischen gesund und günstig, schnell und lecker dominieren. Neben soziokulturellen Gewohnheiten ist vor allem der räumliche Zugang (im Sinne von Nähe) bedeutend für die Wahl des Essens - und zwar bedeutender als der Preis oder die Angebotsqualität und -vielfalt. Wenn es um den Preis geht, wird dieser in Beziehung zum eigenen (gesunden) Körper gesetzt und die verkörperte Gesundheit spielt auch im wechselseitigen Verhältnis zur ,Gesundheit‘ der ökologischen Umweltbedingungen eine Rolle. Diese Verschränkungen zwischen sozialer, verkörperter und ökologischer Umwelt werden eher selten und implizit als Stoff-Wechsel, als transkörperliche Relation thematisiert: Als hybride Verkörperung von Naturkultur (Hybrid hier in Weiterführung von Swyngedouw 2015) von e/Essen, als Wechselwirkungen zwischen ,Natur und ,Kultur'. Insbesondere der zweite Analyseschritt hat ermöglicht, die transkörperliche Relationen zu fokussieren und die Verschränkungen zwischen Körper und Umwelt in beide ,Richtungen“ zu explizieren, als „,[d]as, was man in den Körper reintut und dabei der Umwelt antut" (s.o.) beziehungsweise als das, was man aus der (vergifteten) Umwelt als Essen (Apfel) in den Körper reintut und damit dem Körper antut. 
Wissenschaftliche Voraussetzung dafür ist als ontologische Dimension die Anerkennung der response-ability zwischen Natur und Kultur beziehungsweise die Untrennbarkeit von Naturkultur. Wie Doshi (2017) betont, ist Metabolismus genau dadurch nicht nur metaphorisch wichtig, sondern vor allem als verkörperte Politik interessant, da der Begriff als Prozess sozioökonomisch und -kulturell gerahmte Konsummuster und Routinepraktiken in direkte Wechselwirkung zur biosozialen Verkörperung unterschiedlicher Menschen setzt. Das heißt, im posthumanen Sinne ist Metabolismus ein Prozess, der auf den dynamischen Verschränkungen zwischen allgemein-stofflicher und körperlich-stofflicher Materie basiert und in dem Gesellschaftsstrukturen, kulturelle Diskurse und ökologische Umwelten ,verdaut" und biologisch transformiert werden. In diesem Verständnis wird gleichzeitigt berücksichtigt, dass beziehungsweise inwiefern die Lebensmittel-Umgebungen sozioökonomisch produziert sind - als Wissenskontexte sowie als räumliche Kontexte in der Stadt. Ernährung als sozial- und umwelt-beeinflussten Prozess des essens (Praktik) von Essen (Produkt) zu adressieren verweist also darauf, dass e/Essen weit einflussreicher auf den Körper ist als die reine Nährstoffaufnahme und -verarbeitung: Beim essen werden gesellschaftliche Strukturen und Diskurse sowie die soziale und ökologische Umwelten im wahrsten Sinne des Wortes verdaut, also ver-stoff-wechselt. In der Übertragung von Barads Natur-Umschreibung auf Essen sind Lebensmittel also „weder ein leeres Blatt für das freie Spiel sozialer [und diskursiver] Einschreibungen, noch irgendeine vermittelt vorhandene, transparent gegebene „Dingheit“ (Barad 2015: 61). Essen und essen sind vielmehr „vital materialisms“ (Goodman 2016) und ultimative Naturkultur, sie sind als Lebens-Mittel und Lebens-Mittel zu konzipieren und die biosozialen Stoffwechselprozesse des essens verweisen auf die Frage, wie menschliche Materie (Körper) und stoffliche Materie (Essen) im Prozess des Essens verschränkt sind beziehungsweise intra-agieren (Barad 2015). Der von Stacy Alaimo eingeführte Begriff der „transcorporeality“ hilft dabei als ,recognition not just that everything is interconnected but that humans are the very stuff of the emergent, material world" (Alaimo 2010: 20). Ein gesellschaftstheoretisch reflektierter und praktizierter Fokus auf e/Essen bietet damit aussichtsreiche Möglichkeiten, verkörperte politische Ökologien und transkorporeale Umweltbeziehungen offensiver als Elemente des Urbanen zu adressieren.

Dieser Artikel wird durch Mittel im Forschungsprojekt „Relationale Geographien der Ernährung im Spannungsfeld zwischen Privatsache und Politikum“der Fritz Thyssen Stiftung gefördert.

\section{Endnoten}

[1] Zur Unterscheidung zwischen essen als Praktik und Essen als Produkt werden im Titel und im Text auch Substantivierungen klein geschrieben.

[2] Zugespitzt findet sich diese Technologiefixierung in den aktuellen Smart-City-Nachhaltigkeitsvisionen (siehe Swyngedouw 2015; Kaika 2017; Bauriedl/Strüver 2018; Evans et al. 2019). 
[3] Sie illustrieren dies mit Beispielen aus der Zeit nach der Reaktorkatastrophe in Fukushima, Bürgerkriegssituationen in Kolumbien und Schulgärten in den USA (Hayes-Conroy/ Hayes-Conroy 2013, 2015; siehe auch Morgan 2015).

[4] Gefördert durch die Fritz Thyssen Stiftung 2016 bis 2020.

[5] Die Interviewpartner*innen wurden in und vor Supermärkten, Lebensmittel-Discountern und Biomärkten sowie in Cafés und Fast Food Restaurants angesprochen und dann zu einem ,Eat-along-Interview` in ein Café eingeladen. Diese Interviewart wurde gewählt, um essen und Essen nicht nur als Routine zu thematisieren, sondern um anhand des Produkts und der Praxis des e/Essens die Narration zu stimulieren (vgl. Wenzl et al. 2019 für ähnliche Überlegungen aus einer gleichwohl strenger an Praktiken orientierten Perspektive).

[6] Interviewzitate dienen dabei als Ankerzitate.

[7] In ähnlicher Weise hat auch Mol (2002) eine Praxeographie entwickelt, um zu untersuchen, wie Phänomene (in ihrem Fall Krankheit) in Praktiken entstehen. Solche Phänomene sind damit keine ontologischen Entitäten, sondern entstehen im Zusammenwirken von menschlichen Körpern, medizinischen Instrumenten und gesellschaftlichen Diskursen.

\section{Autor_innen}

Anke Strüver ist Humangeographin. Sie arbeitet mit dem Fokus auf die städtische Mikroebene zu Verkörperungen, Gesundheit, aktiver Mobilität, Ernährung und Digitalisierung im Kontext von Nachhaltigkeit.

anke.struever@uni-graz.at

\section{Literatur}

Alaimo, Stacy (2010): Bodily Natures: Science, Environment, and the Material Self. Bloomington: Indiana University Press.

Alaimo, Stacy (2014): Thinking as the Stuff of the World. In: O-Zone: A Journal of ObjectOriented Studies 1, 13-21.

Alaimo, Stacy (2016): Exposed: Environmental Politics and Pleasures in Posthuman Times. Minneapolis: University of Minnesota Press.

Alaimo, Stacy / Hekman, Susan (Hg.) (2008): Material Feminisms. Bloomington: Indiana University Press.

Anders, Sascha (2015): Lebensmitteldiscounter und Supermarkt. Untersuchung zu Verkehrseffekten, Einzugsgebieten, Vorlieben der Kunden und zum Genehmigungsprozess vor dem Hintergrund der Regelungen des § 11 Abs. 3 BauNVO. In: Raumforschung und Raumordnung 73/3, 219-232.

Augustin, Hanna (2014): Stadt, Ernährung und soziale Ungleichheit: Zur Übertragbarkeit des food desert-Konzeptes auf den deutschen Kontext. Bremen: Universität Bremen, Forschungszentrum Nachhaltigkeit.

Barad, Karen (2003): Posthumanist Performativity: Toward an Understanding of How Matter Comes to Matter. In: Signs: Journal of Women in Culture and Society 28/3, 801-831.

Barad, Karen (2007): Meeting the Universe Halfway. Quantum Physics and the Entanglement of Matter and Meaning. Durham: Duke University Press.

Barad, Karen (2010): Quantum Entanglements and Hauntological Relations of Inheritance: Dis/continuities, SpaceTime Enfoldings, and Justice-to-Come. In: Derrida Today 3/2, 240-268.

Barad, Karen (2012): Agentieller Realismus. Über die Bedeutung materiell-diskursiver Praktiken. Berlin: Suhrkamp.

Barad, Karen (2014): Diffracting Diffraction: Cutting Together-Apart. In: parallax 20/3, 168-187.

Barad, Karen (2015): Verschränkungen. Berlin: Merve Verlag. 
Bauriedl, Sybille (2016): Politische Ökologie: nicht-deterministische, globale und materielle Dimensionen von Natur/Gesellschaft-Verhältnissen. In: Geographica Helvetica 71/4, 341-351.

Bauriedl, Sybille / Strüver, Anke (Hg.) (2018): Smart City - kritische Perspektiven auf die Digitalisierung in Städten. Bielefeld: transcript.

Bozalek, Vivienne / Zembylas, Michalinos (2017): Diffraction or reflection? Sketching the contours of two methodologies in educational research. In: International Journal of Qualitative Studies in Education 30/2, 111-127.

Braidotti, Rosa (2019): A Theoretical Framework for the Critical Posthumanities. In: Theory, Culture and Society 36/6, 31-61.

Carpiano, Richard (2009): Come take a walk with me: The Go Along interview as a novel method for studying the implications of place for health and well-being. In: Health \& Place 15/1, 263-272.

Del Casino, Vincent J. (2015): Social geography I. In: Progress in Human Geography 39/6, 800-808.

Doshi, Sapana (2017): Embodied urban political ecology: five propositions. In: Area 49/1, 125-128.

Elmhirst, Rebecca (2015): Feminist political ecology. In: Tom Perreault / Gavin Bridge / James McCarthy (Hg.): The Routledge Handbook of Political Ecology. London: Routledge, 519-530.

Evans, David (2018): Rethinking material cultures of sustainability: Commodity consumption, cultural biographies and following the thing. In: Transactions of the Institute of British Geographers 43/1, 110-121.

Evans, James / Karvonen, Andrew / Luque-Ayala, Andres / Martin, Chris / McCormic, Kes / Raven, Rob / Voytenko Plagan, Yuliya (2019): Smart and sustainable cities? Pipedreams, practicalities and possibilities. In: Local Environment 24/7, 557-564.

Federici, Silvia (2015): Aufstand aus der Küche. Münster: edition assemblage.

Fox, Nick J. / Alldred, Pam (2018): Mixed methods, materialism and the micropolitics of the research-assemblage. In: International Journal of Social Research Methodology 21/2, 191-204.

Frost, Samantha (2016): Biocultural Creatures: Toward A New Theory of the Human. Durham: Duke University Press.

Galt, Ryan (2013): Placing Food Systems in First World Political Ecology: A Review and Research Agenda. In: Geography Compass 7/9, 637-658.

Gesing, Friederike / Amelang, Katrin / Flitner, Michael / Knecht, Michi (2018): NaturenKulturen-Forschung - Eine Einleitung. In: Dies. (Hg.): NaturenKulturen: Denkräume und Werkzeuge für neue politische Ökologien. Bielefeld: transcript, S. 17-50.

Goodman, Michael K. (2016): Food geographies I: Relational foodscapes and the busy-ness of being more-than-food. In: Progress in Human Geography 40/2, 257-266.

Guthman, Julie (2012): Opening Up the Black Box of the Body in Geographical Obesity Research: Toward a Critical Political Ecology of Fat. In: Annals of the AAG 102/5, 951-957.

Guthman, Julie / Mansfield, Becky (2013): The implications of environmental epigenetics: A new direction for geographic inquiry on health, space, and nature-society relations. In: Progress in Human Geography 37/4, 486-504.

Haraway, Donna (1992): Promises of Monster: A Regenerative Politics for Inappropriate/d Others. In: Lawrence Grossberg / Cary Nelson,/ Paula A. Treichler (Hg.), Cultural Studies. London: Routledge, 295-336.

Haraway, Donna (1995): Die Neuerfindung der Natur. Frankfurt am Main: Campus.

Haraway, Donna (1997): Modest_Witness@Second_Millenium.FemaleMan@C_Meets_On coMouse $^{\mathrm{TM}}$ : Feminism and Technoscience. London: Routledge.

Haraway, Donna (2000): How Like a Leaf. An Interview with Thyrza Goodeve. New York: Routledge.

Haraway, Donna (2008): When Species Meet. Minneapolis: University of Minnesota Press.

Haraway, Donna (2016): Staying with the Trouble: Making Kin in the Chthulucene. Durham: Duke University Press.

Hawkins, Roberta / Ojeda, Diana / Asher, Kiran / Baptiste, Brigitte / Harris, Leila / Mollett, Sharlene / Nightingale, Andrea / Rocheleau, Dianne / Seager, Joni / Sultana, Farhana (2011), A Discussion. In: Environment and Planning D: Society and Space 29/2, 237-253. 
Hayes-Conroy, Jessica / Hayes-Conroy, Allison (2013): Veggies and visceralities: A political ecology of food and feeling. In: Emotion, Space and Society 6, 81-90.

Hayes-Conroy, Allison / Hayes-Conroy, Jessica (2015): Political ecology of the body: a visceral approach. In: Raymond L. Bryant (Hg.), The international handbook of political ecology. Cheltenham (UK) u. a.: Edward Elgar Publishing, 659-672.

Heynen, Nik / Kaika, Maria / Swyngedouw, Erik (Hg.) (2006): In the Nature of Cities. Urban Political Ecology and the Politics of Urban Metabolism. London: Routledge.

Heynen, Nik (2018): Urban political ecology III: The feminist and queer century. In: Progress in Human Geography 42/3, 446-452.

Kaika, Maria (2017): „Don’t call me resilient again!“: the New Urban Agenda as immunology ... or ... what happens when communities refuse to be vaccinated with „smart cities“ and indicators. In: Environment and Urbanization 29/1, 89-102.

Kaika, Maria / Swyngedouw, Erik (2011): The Urbanization of Nature: Great Promises, Impasse, and New Beginnings. In: Gary Bride / Sophie Watson (Hg.), The New Blackwell Companion to the City. Oxford: Blackwell, 96-107.

Krüger, Timmo / Strüver, Anke (2018): Narrative der guten Ernährung': Ernährungsidentitäten und die Aneignung öffentlicher Nachhaltigkeitsdiskurse durch Konsument*innen. In: Zeitschrift für Wirtschaftsgeographie 62/3-4, 217-232.

Landecker, Hannah (2011): Food as exposure: Nutritional epigenetics and the new metabolism. In: BioSocieties 6/2, 167-194.

Lenz Taguchi, Hillevi (2012): A diffractive and Deleuzian approach to analyzing interview data. In: Feminist Theory 13/3, 265-281.

Lenz Taguchi, Hillevi / Palmer, Anna (2013): A more „livable“ school? A diffractive analysis of the performative enactments of girls' ill-/well-being with(in) school environments. In: Gender and Education 25/6, 671-687.

Lorimer, Jamie (2017): Probiotic Environmentalities: Rewilding with Wolves and Worms. In: Theory, Culture \& Society 34/4, 27-48.

Marquardt, Nadine / Strüver, Anke (2018): Körper. Machtgeladene Intra-aktionen zwischen Biologischem und Sozialem. In: Anne Vogelpohl / Boris Michel / Henrik Lebuhn / Johanna Hoerning / Bernd Belina (Hg.), Raumproduktionen II. Theoretische Kontroversen und politische Auseinandersetzungen. Münster: Westfälisches Dampfboot, 38-59.

Mayring, Philip (2014): Qualitative content analysis: Theoretical Foundation, Basic Procedures and Software Solution. https://nbn-resolving.org/urn:nbn:de:0168ssoar-395173 (letzter Zugriff am 11.3.2020).

Mol, Annemarie (2002): The Body Multiple: Ontology in Medical Practice. Durham: Duke University Press.

Mol, Annemarie (2008): I Eat an Apple: On Theorizing Subjectivities. In: Subjectivity 22, 28-37.

Mol, Annemarie (2009): GOOD TASTE: The embodied normativity of the consumer-citizen. In: Journal of Cultural Economy 2/3, 269-283.

Mollett, Sharlene / Faria, Caroline (2013): Messing with gender in feminist political ecology. In: Geoforum 45, 116-125.

Mollett, Sharlene / Faria, Caroline (2018): The spatialities of intersectional thinking: fashioning feminist geographic futures. In: Gender, Place \& Culture 25/4, 565-577.

Morgan, Kevin (2015): Nourishing the city: The rise of the urban food question in the Global North. In: Urban Studies 52/8, 1379-1394.

Romero, Adam / Guthman, Julie / Galt, Ryan / Huber, Matt / Mansfield, Becky / Sawyer, Suzana (2017): Chemical Geographies. In: GeoHumanities 3/1, 158-177.

Schuster, Nina / Höhne, Stefan (2017): Stadt der Reproduktion. Einführung in den Themenschwerpunkt. In: sub \urban. zeitschrift für kritische stadtforschung 5/3, 9-22.

Shillington, Laura (2013): Right to food, right to the city: Household urban agriculture, and socionatural metabolism in Managua, Nicaragua. In: Geoforum 44, 103-111.

Shannon, Jerry (2014): Food deserts: Governing obesity in the neoliberal city. In: Progress in Human Geography 38/2, 248-266.

Sonnino, Roberta (2016): The new geography of food security: exploring the potential of urban food strategies. In: The Geographical Journal 182/2, 190-200.

Strüver, Anke (2012): Fit oder fett - Körperkult(-ur) und die Erforschung der Interdependenzen sozialer und räumlicher Kategorisierungen. In: Geographische Zeitschrift 100/1, $17-33$. 
Strüver, Anke (2019): Von der Inkorporierung und Verkörperung des Sozialen zur Somatisierung der Umwelt: Posthumanistische Überlegungen zum biosozialen Subjekt. In: Geographica Helvetica 74/2, 223-233.

Swyngedouw, Erik (2006): Metabolic urbanization: the making of cyborg cities. In: Nik Heynen / Maria Kaika / Erik Swyngedouw (Hg.), In the Nature of Cities. Urban political ecology and the politics of urban metabolism. London: Routledge, 20-40.

Swyngedouw Erik (2015) Urbanization and environmental futures. In: Tom Perreault / Gavin Bridge / James McCarthy (Hg.): The Routledge Handbook of Political Ecology. London: Routledge, 609-619.

Swyngedouw, Erik / Kaika, Maria (2014): Urban Political Ecology. Great Promises, Deadlock... and New Beginnings? In: Documents d'Anàlisi Geogràfica 6o/3, 459-481.

Tornaghi, Chiara (2014): Critical geography of urban agriculture. In: Progress in Human Geography 38/4, 551-567.

Tornaghi, Chiara (2017): Urban Agriculture in the Food-Disabling City: (Re)defining Urban Food Justice, Reimagining a Politics of Empowerment. In: Antipode 49/3, 781-801.

Ulmer, Jasmine B. (2017): Posthumanism as research methodology: Inquiry in the Anthropocene. In: International Journal of Qualitative Studies in Education 30/9, 832-848.

Warde, Alan (2013): What sort of practice is eating? In: Elizabeth Shove / Nicola Spurling (Hg.), Sustainable Practices: Social theory and climate change. New York/London: Routledge, 17-30.

Warde, Alan (2014): After taste: Culture, consumption and theories of practice. In: Journal of Consumer Culture 14/3, 279-303.

Warde, Alan (2016): The practice of eating. Cambridge: Polity Press.

Warin, Megan (2015): Material Feminism, Obesity Science and the Limits of Discursive Critique. In: Body \& Society 21/4, 48-76.

Wenzl, Christine / Cosima, Werner / Molitor, Katharina / Hornung, Madlen / Rominger, Sarah / Faller, Fabian (2019): Soziale Praktiken in der Forschungspraxis: Empirisch forschen mit Schatzkis site ontology. In: Susann Schäfer / Jonathan Everts (Hg.), Handbuch Praktiken und Raum: Humangeographie nach dem Practice Turn. Bielefeld: transcript, 341-360.

\section{Urban Metabolisms: Embodied Political Ecologies of Food}

Within the last decade, food (as product) and food consumption (as practice) have become increasingly acknowledged as relevant topics in urban spaces of the Global North, both in terms of social relevance and academic recognition. Food and food choices are integral to everyday routines and refer to social and embodied biosocial injustices. Building on Sapana Doshi's proposals for an "embodied urban political ecology", this article focuses on the human body and embodiment in order to address transcorporeal relations between city and ecology through socio-economically and culturally influenced access to good food and the biosocial materialization processes of food consumption. 